\title{
Diseño y construcción de una bicicleta para cargar eléctricamente un teléfono celular
}

\section{Design and construction of a bicycle to electrically charge a cellphone}

\author{
AVILES-FERRERA, José Josíasł*, AMBRIZ-COLIN, Fernando, FLORES-PÉREZ, José Manuel y \\ CANO-RAMÍREZ, Jaime
}

Universidad Tecnológica del Suroeste de Guanajuato, Ingeniería en Mantenimiento Industrial. Carretera Valle-Huanímaro km 1.2, Valle de Santiago, Gto.

ID $1^{\text {er }}$ Autor: José Josías, Aviles-Ferrera / ORC ID: 0000-0003-3503-0819, CVU CONACYT ID: 242164

ID $1^{\text {er }}$ Coautor: Fernando, Ambriz-Colin / ORC ID: 0000-0002-4871-418X, Researcher ID Thomson: R-3696-2018, arXiv Author ID: fambrizc, CVU CONACYT ID: 169757

ID $2^{\text {do }}$ Coautor: José Manuel, Flores-Pérez / ORC ID: 0000-0002-1110-9365, Researcher ID Thomson: R-2163-2018, arXiv Author ID: jose_flores, CVU CONACYT ID: 388269

ID $3^{\text {er }}$ Coautor: Jaime, Cano-Ramírez / ORC ID: 0000-0001-9029-3350, Researcher ID Thomson: Q-8940-2018, arXiv Author ID: JaimeCanoRamirez, CVU CONACYT ID: 341820

DOI: $10.35429 / J I D .2019 .7 .3 .8 .13$

Recibido 05 Marzo, 2019; Aceptado 30 de Junio, 2019

\section{Resumen}

En este proyecto se realizó un estudio, diseño y construcción de un dispositivo de generación de energía eléctrica. Se produce energía eléctrica, mediante el movimiento de un generador eléctrico, suficiente para recargar dispositivos móviles a través del pedaleo de una bicicleta estática. De la investigación realizada sobre la generación de la energía eléctrica resultó que un generador con las características adecuadas puede proporcionar la energía necesaria y suficiente para alimentar eléctricamente un teléfono móvil. En este artículo se muestra la investigación, procedimiento de diseño, detalle y fabricación del dispositivo generador de electricidad. Este proyecto beneficiará a todas las personas en general, ya que cuando requieran cargar su teléfono celular podrán pedalear una bicicleta estática y cargar eléctricamente su celular. Con el ejercicio que realicen al pedalear la bicicleta bajarán de peso, con lo cual se obtendrán dos beneficios: Cargar el teléfono móvil y hacer ejercicio para estar más saludable.

Generación, Dinamo, Bicicleta

\begin{abstract}
In this project a study, design and construction of an electric power generation device was carried out. Electric power is produced, by the movement of an electric generator, enough to recharge mobile devices through the pedaling of an exercise bike. From the research conducted on the generation of electric power it turned out that a generator with the appropriate characteristics can provide the necessary and sufficient energy to electrically power a mobile phone. This article shows the research, design procedure, detail and manufacturing of the electricity generating device. This project will benefit all people in general, since when they need to charge their cell phone they can pedal an exercise bike and electrically charge their cell phone. With the exercise they do when pedaling the bike they will lose weight, which will give you two benefits: Charge your mobile phone and exercise to be healthier.
\end{abstract}

Generation, Dynamo, Bike

Citación: AVILES-FERRERA, José Josías, AMBRIZ-COLIN, Fernando, FLORES-PÉREZ, José Manuel y CANORAMÍREZ, Jaime. Diseño y construcción de una bicicleta para cargar eléctricamente un teléfono celular. Revista del Diseño Innovativo. 2019. 3-7: 8-13

\footnotetext{
* Correspondencia del Autor (Correo electrónico: jjavilesfe@utsoe.edu.mx)

$\dagger$ Investigador contribuyendo como primer autor.
} 


\section{Introducción}

Este proyecto nace en Valle de Santiago, Guanajuato, a través de la necesidad de obtener energía eléctrica a partir del esfuerzo físico. Esta energía será aprovechada para la recarga de celulares. Dicho sistema es apropiado para áreas de deporte o áreas sociales, en donde las personas puedan recurrir a este sistema y cargar sus teléfonos móviles. Este proyecto toma en cuenta que la mayoría de las personas cuentan con un teléfono celular y estos equipos requieren energía eléctrica. Este proyecto también está diseñado para hacer que las personas realicen más actividad física y que tengan una vida saludable.

Este dispositivo está diseñado para que las personas hagan más ejercicio, al mismo tiempo que se logra generar energía eléctrica de una manera limpia, sin contaminantes, con lo cual se beneficia a la población y al medio ambiente. De esta manera, se piensa en concientizar a las personas a realizar la activación física y que mantengan una vida saludable.

\section{Justificación}

Actualmente los avances tecnológicos han hecho que la vida del hombre sea más cómoda y menos desgastante físicamente. La energía física se desperdicia ya que no se tiene el hábito de aprovecharla. Por otro lado, el hombre busca nuevas formas $\mathrm{y}$ alternativas para generar energía eléctrica. Si bien son conocidas las nuevas tecnologías de generación por el sol o el aire, estas tecnologías son costosas. Por lo que es necesario buscar nuevas fuentes alternativas de energía que sean más baratas y mejor aún, que se pueda aprovechar la energía propia almacenada, (Osorio, 2007).

\section{Objetivo General}

La idea consiste en diseñar una bicicleta estática generadora de energía eléctrica para la recarga eléctrica de celulares y así poder prevenir la obesidad y concientizar a las personas a que realicen más actividad física, de esta manera se aprovecharía nuestra propia energía.

Existen proyectos similares en la actualidad. Pero, la principal diferencia de este proyecto respecto a los anteriores, es la aplicación que se le da, la cual consiste en la recarga de un teléfono móvil.
El alcance de este proyecto abarca desde la investigación bibliográfica, el diseño conceptual, así como la ingeniería básica y de detalle, los costos del prototipo y de fabricación. Se consideró desde el diseño e ingeniería conceptual hasta la fabricación, ensamble y pruebas del prototipo.

\section{Marco Teórico}

La generación de energía eléctrica, se produce en alternadores o generadores, en términos generales, consiste en transformar alguna clase de energía, ya sea esta química, mecánica, térmica o luminosa, entre otras, en energía eléctrica. De todos los tipos de energía, la que mayor uso ha recibido es la energía eléctrica. Esta popularidad está dada por su difusión tanto en la economía de las naciones como en el uso doméstico. La energía eléctrica es un insumo primario para la realización de las actividades productivas, de transformación y servicios en el país. La energía eléctrica tiene las cualidades de ser de fácil generación y distribución hasta los consumidores. También, por su fácil transformación a otras formas de energía, tales como: la térmica, luminosa, mecánica, entre otras. El rápido desarrollo del aprovechamiento de la energía eléctrica estimuló a la construcción de grandes plantas generadoras capaces de convertir la energía mecánica, obtenida mediante otras fuentes de energía primaria, en energía eléctrica, constituyendo el primer escalón del sistema de suministro eléctrico.

Las máquinas que producen corriente eléctrica para su uso en gran escala se llaman generadores. El principio del generador es simple, cada vez que un conductor se mueve cerca del extremo de un imán se origina en él una diferencia de tensión eléctrica (voltaje). Esta es una notable propiedad del magnetismo, es decir, la capacidad de crear un flujo de electrones.

La Ley de Faraday habla acerca de la variación del campo magnético, cuando éste cambia produce un campo eléctrico que se representa en una fuerza electromotriz (FEM), como existe un campo eléctrico aparecerá una corriente, todo debido al campo magnético, (Kosow, 2012). El campo magnético es la zona que rodea a un imán y en el cual se manifiestan sus efectos. Los campos magnéticos se representan habitualmente mediante líneas llamadas líneas de fuerza que van de polo norte (N) al polo sur (S) de un imán, como se representa en la Figura 1.

AVILES-FERRERA, José Josías, AMBRIZ-COLIN, Fernando, FLORES-PÉREZ, José Manuel y CANO-RAMÍREZ, Jaime. Diseño y construcción de una bicicleta para cargar eléctricamente un teléfono celular. Revista del Diseño Innovativo. 2019 


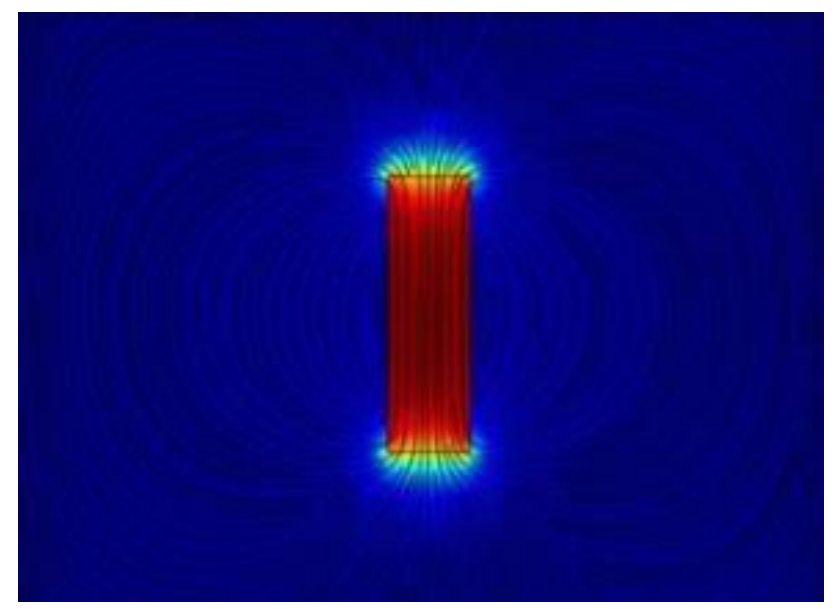

Figura 1 Campo magnético

El voltaje inducido en un circuito va a ser igual a la rapidez con la que varía el campo magnético. Para generar electricidad se necesita variar el campo magnético en un conductor de corriente, en este caso será una bobina. Al variar el campo magnético (imán) cerca de un alambre de cobre u otro conductor, éste se verá afectado por la (FEM) y generará electricidad.

El campo magnético inducido por el imán debe estar en movimiento a través de la bobina para generar una fuerza electromotriz, al introducir el imán dentro del campo magnético se notará como la fuerza electromotriz inducida llega al punto más alto, cuando el imán pasa por en medio de la bobina se registrará un cambio de signo en el voltaje, cuando el imán sale de la bobina se registrará un voltaje opuesto en la dirección inversa a la del imán moviéndose hacia la bobina, como se ilustra en la figura 2 .

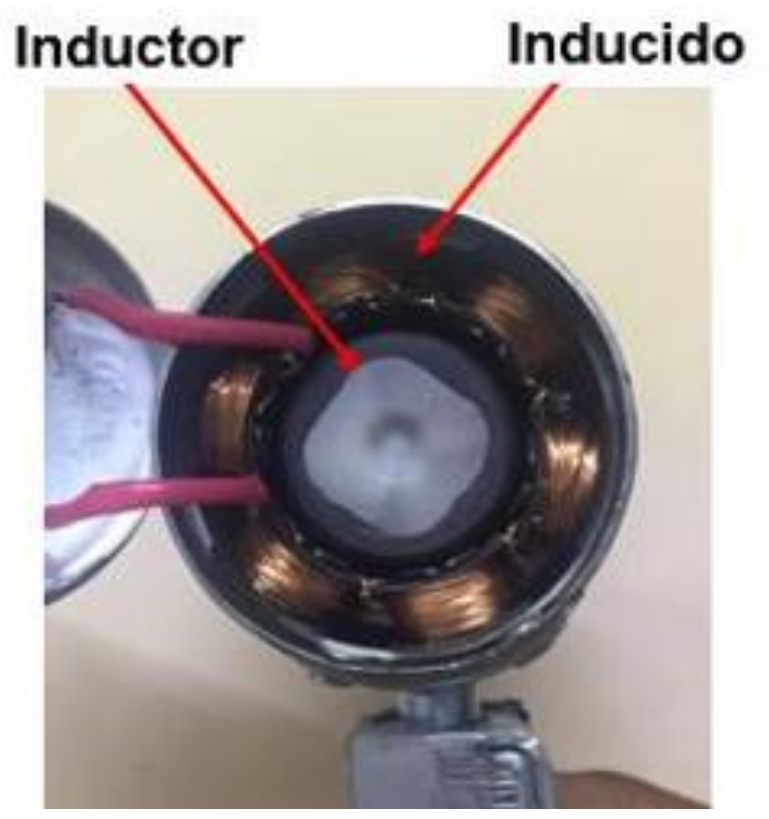

Figura 2 Fuerza electromotriz inducida
La energía electromagnética debida a las fuerzas eléctricas y magnéticas del átomo se relaciona con la fuerza mecánica que se aplica a la materia y al movimiento. De esta manera, la energía mecánica se puede convertir en energía eléctrica y viceversa mediante sistemas dinámicos (dínamos). Así, la energía mecánica de una fuerza aplicada a un eje a través de una bicicleta y trasmitida por la cadena se convierte fácilmente en energía eléctrica mediante un alternador, la energía que se produce se transforma por conversión de energía electromagnética a un mayor voltaje para su trasmisión a grandes distancias y en un punto terminal, se transforma una vez más para su distribución y así poder utilizar esa energía generada. Esta energía puede convertirse en carga eléctrica para dispositivos celulares.

Un dinamo de bicicleta es un dispositivo mecánico que genera electricidad a partir de la energía rotatoria que actúa sobre la rueda de la bicicleta. Normalmente, la pieza móvil es el imán y la pieza fija es la del arrollamiento, aunque puede ser al revés. El imán se mueve solidario con el eje que en su extremo tiene la corona que se apoya sobre el neumático. En los dinamos de buje, el eje de la rueda es el eje solidario al imán. En definitiva, en una dinamo una parte del generador rota (el rotor) y la otra parte permanece inmóvil (el estator). El rotor se compone de imanes permanentes de un cierto tipo y el estator se compone de bobinas de alambre.

El campo magnético del rotor se transfiere a las bobinas del estator cada vez que da una vuelta entera e induce la electricidad que se vierte a través del cableado hacia un dispositivo es capaz de aprovecharla, en este caso son los dispositivos electrónicos. Estos dispositivos deberán adaptarse al voltaje y potencia del dinamo. La electricidad producida podría utilizarse para la iluminación de la bicicleta, pero en este caso lo que se quiere es aprovecharla para la carga de dispositivos electrónicos.

Los dinamos de botella son los que funcionan mediante el rozamiento de la cabeza del dinamo con el neumático de la rueda de la bicicleta. Con ellos se puede conseguir la recarga de aparatos electrónicos mientras se pedalea, aunque no sean lo suficientemente eficientes. Como principales ventajas se tienen que son ligeros y baratos. 
Sin embargo, tiene una serie de desventajas. Su principal problema es que su localización las hace muy vulnerables a la suciedad y a la humedad, (Valencia, 2017).

\section{Desarrollo}

Inicialmente, mediante la investigación, se obtuvo el siguiente diagrama, Figura 3, en donde se muestra el sistema con los 4 componentes, la estructura, en donde se compone toda la bicicleta, alternador, aquí es donde se generará la energía a utilizar, rectificador, aquí es donde se medirá la energía para obtener la necesaria y suministrarla al teléfono móvil y por último el acumulador, en donde se almacenará la energía.

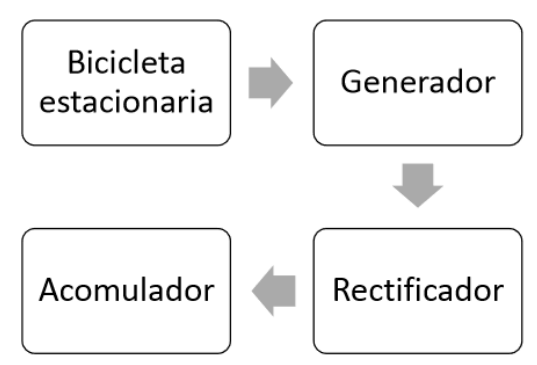

Figura 3 Sistema general

Se realizaron pruebas con un dinamo el cual tiene por característica una entrega de $5.5 \mathrm{~W}$ y 12 Volts de voltaje. Para saber la corriente que genera un dinamo de botella se empleó la ecuación (1):

$P=V I$ ecuación (2):

Despejando la ecuación (1) se obtiene la

$$
\begin{aligned}
& I=\frac{P}{V} \\
& \mathrm{P}=5.5 \mathrm{~W} \\
& \mathrm{~V}=12 \mathrm{~V} \\
& I=5.5 \mathrm{~W} / 12 \mathrm{~V}=0.458 A \\
& I=458 \mathrm{~mA}
\end{aligned}
$$

Para el desarrollo del circuito rectificador se utilizó un programa llamado: "Proteus". Ahí, se simulan los resultados del circuito mediante la realización del diagrama electrónico. Este circuito tiene la capacidad de rectificar el voltaje producido de un dinamo de $5 \mathrm{~V}$ a $12 \mathrm{~V}$, y mantener el Amperaje resultante del propio dinamo. La figura 4 muestra el circuito con sus elementos electrónicos.

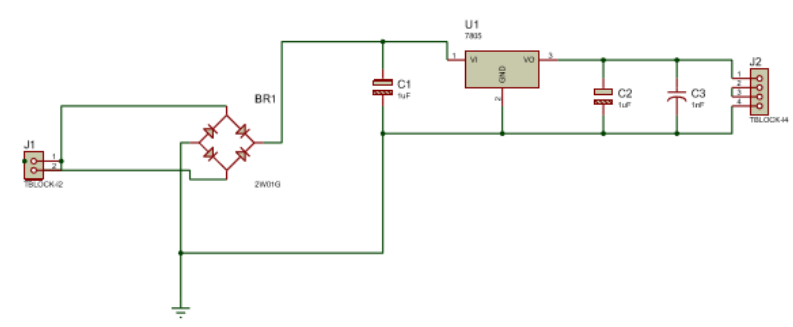

Figura 4 Circuito rectificador en Proteus

Los elementos se conectaron a través de una plantilla de prueba y se verificó el funcionamiento del circuito electrónico. La figura 5 muestra la interconexión de todos los elementos.

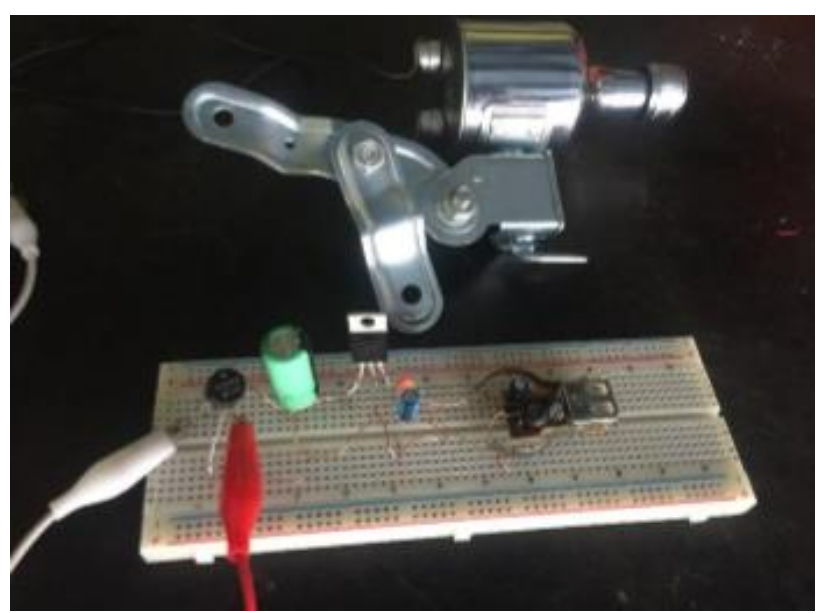

Figura 5 Conexión del circuito regulador y rectificador

Se realizaron pruebas con el circuito completo conectado, es decir, bicicleta, dinamo, circuito rectificador-alimentador y un teléfono celular. Se conectó la salida del dinamo que es corriente alterna a las patas de entrada del puente de diodos rectificador, de ahí es convertida a corriente directa, después rectificada a 5 Volts y por último se conecta una salida USB (Universal Serial Bus) hembra. Para conectar un teléfono celular y alimentar la batería del mismo con la suficiente energía generada por el dínamo.

Además de generar energía eléctrica para cargar un celular a través de la bicicleta, de forma adicional, se desarrolló un sistema que mide las calorías quemadas al hacer ejercicio por el pedaleo de la bicicleta y convertidas en energía eléctrica. También, se implementó un dispositivo que mide la distancia recorrida en kilómetros. Para desarrollar e implementar esta parte, se realizaron las siguientes consideraciones: 
La velocidad de una persona que hace ejercicio en una bicicleta estática en promedio es de 90 a $100 \mathrm{rpm}$ (revoluciones por minuto), si se cuentan las vueltas que gira la rueda de la bicicleta estática en un periodo de 1 minuto, a través de un sensor infrarrojo y utilizando una tarjeta Arduino para hacer los cálculos, se obtiene la velocidad promedio del pedaleo del usuario en la bicicleta.

Para obtener los datos de los sensores y realizar los cálculos pertinentes se desarrollaron códigos en la plataforma Arduino. A través de un sensor infrarrojo el cual mide las revoluciones de la llanta de la bicicleta. Se obtiene el peso de la persona, por medio de las dos celdas de carga. Una vez obtenidos estos datos, peso, velocidad, distancia y tiempo, se procedió a realizar una interfaz en LabView en donde se muestran los resultados de las ecuaciones, así como las gráficas del comportamiento de los mismos.

De esta manera, se conocen el total de las calorías quemadas y los kilómetros recorridos al hacer ejercicio en la bicicleta estática. Los datos son almacenados, procesarlos y mostrarlos en gráficas de esta manera se puede conocer el progreso que tiene cada persona al mejorar su salud física, tal como se muestra en la Figura 6.

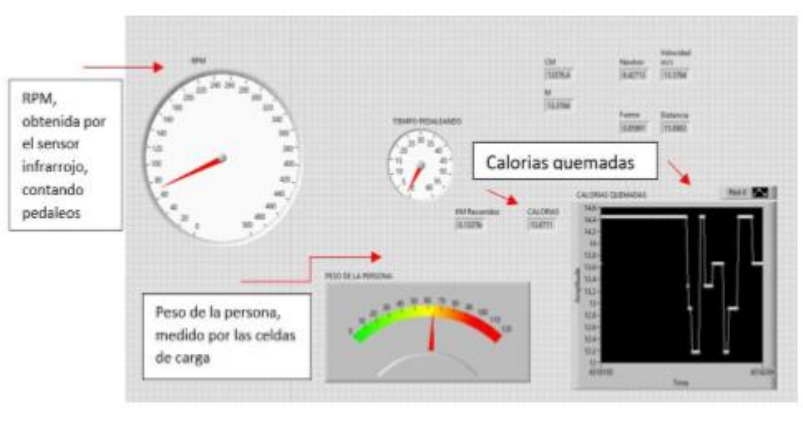

Figura 6 Graficas representativas de LabVIEW

\section{Resultados}

Se realizaron diferentes pruebas con diferentes motores y generadores. La mejor opción fue utilizar un dinamo generador de 12 Volts y 5.5 $\mathrm{W}$, de esta manera se comprobó que se puede generar suficiente voltaje y suficiente corriente para alimentar un teléfono celular mientras se está pedaleando. En la figura 7, se muestra el resultado final que se obtuvo al integrar todos los sistemas en la bicicleta, lo que resulta un prototipo de prueba con un circuito para medir de número de revoluciones, con celdas de carga para medir el peso de la persona y un último para la regular el voltaje.
También, se muestra el dinamo instalado con el Arduino para adquirir los datos y en la parte superior, para colocar el teléfono celular, un soporte para celulares y un cable USB con varias salidas para diferentes tipos de teléfonos celulares.

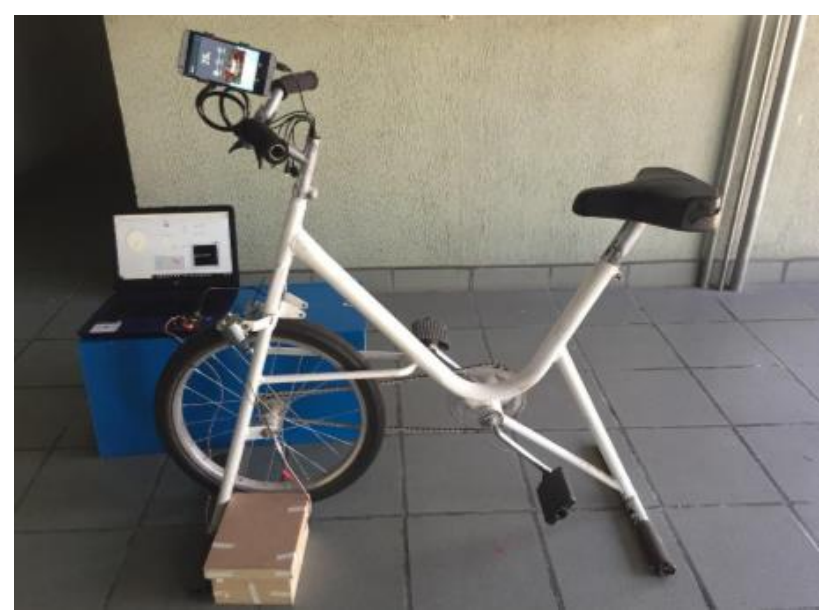

Figura 7 Prototipo de prueba

De esta manera se obtuvo el resultado querido con las características y objetivos mencionados anteriormente, una bicicleta estática generadora de energía eléctrica para cargar un teléfono móvil. La figura 8 muestra el prototipo final con todos los aditamentos desarrollados, los cuales son: El medidor de velocidad y distancia, el medidor de la cantidad de calorías quemadas, el sistema de generadores mediante un arreglo de dinamos que proporciona la corriente y el regulador de voltaje que rectifica y regula el voltaje necesario para cargar eléctricamente un teléfono celular.

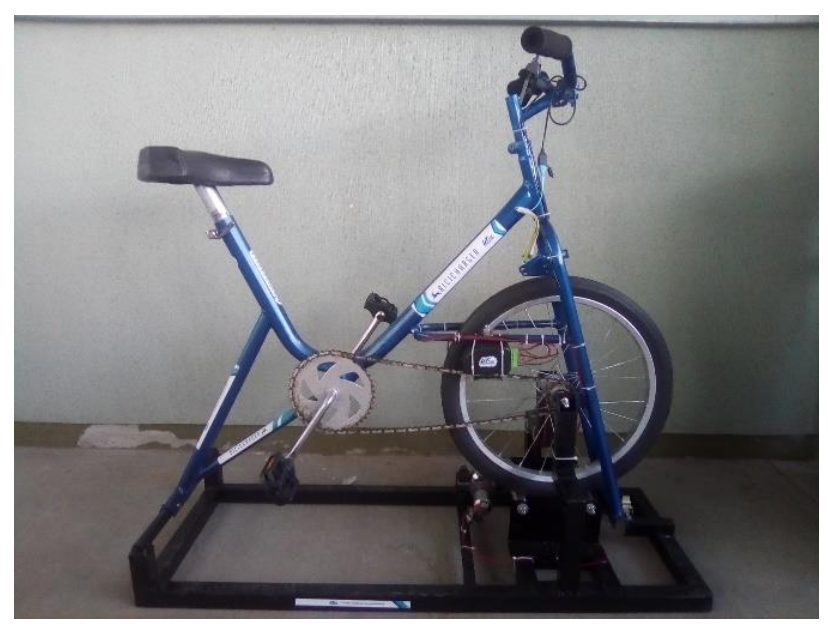

Figura 8 Prototipo final 


\section{Conclusiones}

Se demuestra que es posible generar energía eléctrica mediante la conversión de la energía mecánica, a partir del movimiento de un eje que transmite la energía que se produce por la fuerza aplicada por el pedaleo de una bicicleta y que se transmite a través de la cadena o polea y mueve un pequeño generador eléctrico. Aplicando la ley de la conservación de la energía, "La energía no se crea ni se destruye, sólo se transforma de una forma a otra, sin que cambie la cantidad total de energía", se obtiene un mecanismo que convierte la energía acumulada en el cuerpo de una persona como grasa, en energía eléctrica, a través del ejercicio provocado por el pedaleo de una bicicleta estática y cuya energía generada se usa para cargar eléctricamente un teléfono celular.

De esta manera se obtiene un doble beneficio, ya que, al ejercitarse continuamente una persona mediante el pedaleo de una bicicleta estática, hace que queme calorías, es decir, grasa y reduzca el exceso de energía cumulada en su cuerpo. Además, esa energía no se elimina y por ende se desperdicia, sino que se convierte en una energía útil para una aplicación específica, la cual consiste en alimentar un teléfono celular.

Al finalizar este proyecto se obtuvo un prototipo capaz de generar energía eléctrica mediante el ejercicio que hace una persona al pedalear una bicicleta estática, con lo cual elimina la energía almacenada en su cuerpo en forma de grasa, esta energía que se elimina se puede medir de tal manera que conoce la cantidad de calorías que pierde una persona y al mismo tiempo, el kilometraje que recorre al pedalear la bicicleta. Además, puede usar esa energía que ya no tiene más en su organismo para cargar eléctricamente su teléfono celular.

\section{Referencias}

Anaya, G. y Juárez, X. (2003). "Utilización de fuerza mecánica para generar electricidad". Universidad Autónoma del Estado de Hidalgo.

Boylestad, R. y Nashelsky, L. (1998). "Fundamentos de la electricidad". Prentice-Hall, Hispanoamericana, S.A.

Irving, L. y Kosow Ph. (2012). "Máquinas Eléctricas y Transformadores". Revista Electrical and Computer Engineering Technology Southern Collage of Technology.
Osorio, A. (2007). “Generador eléctrico accionado por fuerza humana", Revista Academia de Ingeniería Mecánica Eléctrica, Universidad Juárez Autónoma de Tabasco.

Peña, M. y Bacallao, J. (2000). "La obesidad en la pobreza". Organización Panamericana de la Salud. Washington D. C.

Romero, J. (2000). “La electricidad”. Centro de capacitación profesional.

Valencia, A. (2017). "Diseño de rodillo universal para bicicletas con sistema de recuperación de energía”. Universidad pública de navarra. 